\title{
SPATIAL PATTERNS OF THE TAU PATHOLOGY IN PROGRESSIVE SUPRANUCLEAR PALSY
}

\author{
Richard A. Armstrong ${ }^{*}$ and Nigel J. Cairns ${ }^{2}$ \\ ${ }^{1}$ Vision Sciences, Aston University, Birmingham B4 7ET, UK; 2 Departments of \\ Neurology, and Pathology, and Immunology, Washington University School of \\ Medicine, St Louis, Missouri, USA.
}

*Corresponding Author: R.A. Armstrong, Vision Sciences, Aston University, Birmingham, B4 7ET, UK (Tel: 0121-204-4102; Fax: 0121-204-4048, EMail: R.A. Armstrong@aston.ac.uk).

Running title: Neuropathology of progressive supranuclear palsy 


\begin{abstract}
Objective Progressive supranuclear palsy (PSP) is characterized neuropathologically by neuronal loss, gliosis, and the presence of tau-immunoreactive neuronal and glial cell inclusions affecting subcortical and some cortical regions. The objectives of this study were to determine (1) the spatial patterns of the tau-immunoreactive pathology, viz., neurofibrillary tangles (NFT), oligodendroglial inclusions (GI), tufted astrocytes (TA), and Alzheimer's disease (AD)-type neuritic plaques (NP) in PSP and (2) to investigate the spatial correlations between the histological features. Patients Postmortem material of cortical and subcortical regions of eight PSP cases was studied. Spatial pattern analysis was applied to the NFT, GI, TA, NP, abnormally enlarged neurons (EN), surviving neurons, and glial cells. Results NFT, GI, and TA were distributed either at random or in regularly distributed clusters. The EN and NP were mainly randomly distributed. Clustering of NFT and EN was more frequent in the cortex and subcortical regions respectively. Variations in NFT density were not spatially correlated with the densities of either GI or TA but were positively correlated with the densities of EN and surviving neurons in some regions. Conclusion (1) NFT were the most widespread tau-immunoreactive pathology in PSP being distributed randomly in subcortical regions and in regular clusters in cortical regions, (2) GI and TA were more localized and exhibited a regular pattern of clustering in subcortical regions, and (3) neuronal and glial cell pathologies were not spatially correlated.
\end{abstract}

Key Words: Progressive supranuclear palsy (PSP), Neurofibrillary tangle (NFT), Oligodendroglial inclusion (GI), Tufted astrocyte (TA), Spatial pattern 


\section{Introduction}

Progressive supranuclear palsy (PSP) is a mainly sporadic, multisystem neurodegenerative disorder first described as a clinical entity in 1964 [1]. The disorder has a prevalence of $6.4 / 100000$ of the population [2,3] and is the second most common syndrome in which 'parkinsonism' is a prominent symptom [4]. The first description of PSP was based on nine cases that exhibited supranuclear ophthalmoplegia, pseudobulbar palsy, dysarthria, and dystonic rigidity of the upper neck and trunk [5,6]. In addition, despite the predominantly subcortical symptoms, some patients also exhibit frontal lobe signs ('dysexecutive syndrome') suggesting a degree of cortical involvement $[6,8]$.

Neuropathologically, PSP may be distinguished from normal adult brains and from Parkinson's disease (PD) by symmetrical tissue loss in the frontal cortex (maximal in the orbitofrontal and medial frontal cortices), subcortical nuclei (midbrain, caudate, and thalamic) [9]. In addition, there is neuronal loss, gliosis, neurofibrillary tangles (NFT), granulovacuolar change (GVC), and demyelination, mainly affecting the globus pallidus, subthalamic nucleus, substantia nigra, dentate nucleus of the cerebellum, and brain stem tegmentum [10,11]. The NFT are structurally and biochemically different to those observed in Alzheimer's disease (AD). There are both globose and flame-shaped NFT composed of $15 \mathrm{~nm}$ straight filaments comprising six or more protofilaments $2-5 \mathrm{~nm}$ in diameter $[12,13]$. The NFT are tauimmunoreactive and composed largely of 4-microtubule binding-repeat (4R) tau [14]. PSP is therefore classified among the tauopathies, a group of disorders that also includes AD, Pick's disease (PiD), corticobasal degeneration (CBD), the NFT predominant form of senile dementia (NFT-SD), argyrophilic grain disease (AGD), and parkinsonism-dementia complex of Guam (Guam PDC) [15,16]. Abnormally enlarged neurons (EN) are present, especially in the inferior olivary nucleus [10] and throughout the neocortex [17]. In addition, 'tufted' astrocytes (TA) and glial inclusions (GI) ('coiled bodies') [18-20] are present in the motor cortex and striatum [14]. The presence of TA is often regarded as diagnostic for PSP [19] and distinguishes the disorder from the closely-related CBD [21]. Finally, neuritic plaques (NP), also termed 'NFT-associated neuritic clusters', are present in the hippocampus 
and adjacent gyri of some cases [22] and consist of numerous degenerating axon terminals.

In other tauopathies such as Alzheimer's disease (AD), Pick's disease (PiD), and corticobasal degeneration (CBD), the pathology exhibits a characteristic spatial pattern in coronal sections of brain tissue, viz., in the cortex, the inclusions occur in clusters that exhibit a regular distribution parallel to the pia mater [23]. This topographic pattern suggests that the inclusions develop in association with clusters of neurons associated with the cortico-cortical projections [23-25]. PSP, however, differs from other tauopathies in first, the pathology is predominantly subcortical and second, significantly affects astrocytes and oligodendrocytes as well as neurons. A previous study suggested that NFT may be clustered in some cortical regions in PSP $[7,26]$. Hence, to characterize the pattern of degeneration in cortical and subcortical regions in PSP and the relationships between the various pathological inclusions, we determined: (1) whether the NFT, GI, TA, and NP exhibited a similar topographic distribution to those previously reported in the tauopathies and (2) the spatial correlations between the NFT, GI, TA, and NP and between the tau-immunoreactive inclusions and surviving neurons, EN, and glial cells.

\section{Materials and Methods}

\section{Cases}

Brain material from eight cases of PSP (see Table 1) was obtained from the Department of Neuropathology, Institute of Psychiatry, King's College London, UK and were the same eight cases used to study laminar distribution of the pathology in PSP [8]. Patients had no family history of psychiatric or neurological disorders and were clinically diagnosed as 'definite' PSP using National Institute of Neurological Disorders and Stroke (NINDS) and the Society of PSP (SPSP) clinical criteria [2729]. Two clinical phenotypes of PSP have been identified, viz., Richardson's syndrome (RS) and PSP-parkinsonism (PSP-P), the two subtypes varying in disease duration and in tau isoforms [30]. All cases studied were of the classical RS subtype of PSP. In addition, with two exceptions (Cases C and F), cases represented Braak tangle stages I or II [31]. The most common disease presentations were confusion 
and/or memory problems (four patients) and early falls (three patients). Later, postural instability leading to frequent falls, rigidity, poor memory, and gait problems were more frequent. Memory problems and confusion were exhibited by the majority of patients. Visual problems were present in all patients, vertical supranuclear gaze palsy being the most consistent symptom. A diagnosis of AGD, which also has 4R-tau positive oligodendrocytic and astrocytic inclusions [32], can be excluded as the majority of patients were young, exhibited supranuclear gaze palsy, and EN were mainly absent in the cingulate gyrus and amygdala. A diagnosis of corticobasal degeneration (CBD) was excluded by the absence of astrocytic plaques, the presence of globose or flame shaped NFT rather than wispy-fine NFT, and lack of neuropil threads $[14,33]$. Although EN are present in PSP they are not as abundant as in other tauopathies such as CBD [17] and our cases conform to this distribution. In addition where CBD and PSP pathology occur in the same case, there is the possibility of a diagnosis of frontotemporal dementia with parkinsonism linked to chromosome 17 (FTLD-17) but no MAPT mutations were present in the cases studied.

\section{Histological methods}

After death, the consent of the next of kin was obtained for brain removal, following local Ethical Committee procedures and the 1995 Declaration of Helsinki (as modified Tokyo, 2004). The following tissue blocks were taken from each case: (1) frontal lobe at the level of the genu of the corpus callosum to study the superior frontal cortex (SFC) (B8) and anterior cingulate gyrus (CG) (B24), (2) temporal lobe at the level of the lateral geniculate body (LGN) to study the inferior temporal gyrus (ITG) (B20), sectors CA1/2 of the hippocampus (HC), and dentate gyrus (DG), (3) striatum at the level of the anterior commissure to study the putamen (PT) and globus pallidus (GP), (4) mid-brain at the level of the third cranial nerve to study the substantia nigra (SN) and red nucleus (RN), (5) rostral pons through the mid-point of the locus caeruleus (LC) to study both the LC and pontine nuclei (PN), (6) midmedulla through the maximum diameter of the inferior olive to study the inferior olivary nucleus (ION), and (7) cerebellar hemisphere taken through the superior cerebellar peduncle to include the dentate nucleus. Tissue was fixed in $10 \%$ formal buffered saline and embedded in paraffin wax. $7 \mu \mathrm{m}$ sections were stained with a variety of standard staining methods including $\mathrm{H} / \mathrm{E}$, Bielschowsky silver staining 
method, Luxol fast blue, and glial fibrillary acidic protein (GFAP). In addition, sections were immunolabelled with an antibody (TP70) [28] that recognizes all forms of tau and is raised to the peptide corresponding to residues $428-441$ at the $\mathrm{C}$-terminal portion of the protein.

\section{Morphometric methods}

In cortical regions (SFC, CG, ITG), the histological features were counted along strips of tissue (3200 to $6400 \mu \mathrm{m}$ in length) parallel to the pia mater using $50 \times 250 \mu \mathrm{m}$ sample fields arranged contiguously. The fields were located to study variations in density along the upper (laminae II/III) and lower (laminae V/VI) cortex, the short edge of the field $(50 \mu \mathrm{m})$ being aligned with guidelines marked on the slide and arranged parallel to the pia mater. In the $\mathrm{HC}$, the pathological changes were counted from the beginning of sector CA1 to the CA2/CA3 boundary, the short dimension of the field being aligned with the alveus. In the DG, the field was aligned with the upper edge of the granule cell layer. Two areas were sampled in the striatum, viz., the PT and the GP, the fields being located along the edges of the external and internal capsules respectively. In the midbrain, fields were aligned along the lower edge of the crus cerebri to sample the $\mathrm{SN}$ as far as the cortinonigral fibres and transversely across the maximum diameter of the RN. In the rostral pons, the fields were arranged across the maximum diameter of the $\mathrm{LC}$ and across the $\mathrm{PN}$ at the base of the pons. In the medulla, the fields followed the contours of the principal ION and were aligned with the central tegmental tract. In the cerebellum, the fields were located along the lower edge of the granule cell layer to sample the Purkinje cells. All NFT, GI, TA, NP, EN, surviving neurons, and glial cells were counted within each sample field. GI were associated with oligodendroglial cell nuclei and TA with an astrocyte cell body while NFT were present in the cytoplasm of larger cells with a distinct nucleus and cytoplasm and immunolabelled sections were compared with Bielschowsky silver stain to ensure that immunolabelled cells contained NFT rather than being simply tauimmunoreactive neurons. NP were tau immunoreactive structures consisting of clusters of degenerating axon terminals and silver stained sections were examined to ensure that they were not the astrocytic plaques (AP) characteristic of CBD. EN in PiD, CBD, PSP, and AD may be immunolabelled with anti-phosphorylated neurofilament (NF) protein although EN associated with infarction are less intensely 
labeled by this procedure [34]. In addition, alpha-B-crystallin, a heat shock protein that functions as a molecular chaperone under conditions of stress, is reported to be a sensitive marker for detecting EN in AD [35]. Nevertheless, it is unclear whether all EN are immunolabeled by alpha-B-crystallin. For example, after infarction, normal neurons become convex and develop the characteristic appearance of $\mathrm{BN}$ after about 10 days but alpha-B-crystallin immunoreactivity increases only in the later stages of their development [36]. Small numbers of EN were observed in the PSP cases and hence, a morphological criterion was used to define EN as not all of these cells in PSP were immunoreactive to alpha B-crystallin. Hence, EN had abnormally enlarged perikarya, lacked NFT, had a shrunken nucleus displaced to the periphery of the cell, and the maximum cell diameter was at least three times the nucleus diameter. Surviving neurons were identified as cells containing at least some stained cytoplasm in combination with larger shape and non-spherical outline. Small spherical or asymmetrical nuclei without cytoplasm but with the presence of a thicker nuclear membrane and more heterogeneous chromatin were identified as glial cells.

\section{Data analysis}

Measuring the spatial pattern of a histological object, i.e., whether the object is distributed at random, regularly, or forms clusters, may provide more information than a simple estimate of abundance [37,38]. Hence, the spatial pattern of a pathological lesion such as NFT may reflect the degeneration of underlying neuroanatomical structures and hence, may be useful in determining lesion pathogenesis [37].

To determine the spatial patterns of the histological features, the data were analyzed by spatial pattern analysis [37,38]. This method uses the variance-mean ratio (V/M) of the data to determine whether the histological features were distributed randomly $(\mathrm{V} / \mathrm{M}=1)$, regularly $(\mathrm{V} / \mathrm{M}<1)$, or were clustered $(\mathrm{V} / \mathrm{M}>1)$ along a strip of tissue. Counts of a feature in adjacent sample fields were then added together successively to provide data for increasing field sizes, e.g., 50 × $250 \mu \mathrm{m}, 100 \times 250 \mu \mathrm{m}, 200 \times 250 \mu \mathrm{m}$ etc., up to a size limited by the length of the strip sampled. V/M was plotted against field size measured parallel to the pia mater to determine first, whether the clusters of a histological feature were regularly or randomly distributed and second, to estimate 
the mean cluster size. A V/M peak indicated the presence of regularly spaced clusters while an increase in $\mathrm{V} / \mathrm{M}$ to an asymptotic level suggested the presence of randomly distributed clusters [38]. The statistical significance of a peak was tested using the ' $t$ ' distribution [37,38]. The frequencies of the different types of spatial pattern were compared between cortical and subcortical regions using chi-square $\left(\chi^{2}\right)$ contingency table tests. Spatial correlations between histological features were tested for each region by calculating Pearson's correlation coefficient ('r') between the densities measured parallel to the tissue boundary for each region [39].

\section{Results}

Examples of the tau-immunoreactive pathology in PSP are shown in Figs 1 and 2. NFT (Fig 1) were present in cells with a distinct nucleus and cytoplasm while TA (Fig 2) consisted of tau immunoreactive processes clearly associated with an astrocyte cell body.

Examples of the spatial patterns exhibited by the histological features in PSP are shown in Fig 3. The NFT (Case B, CG) exhibited a V/M peak at a field size of $400 \mu \mathrm{m}$ suggesting the presence of regularly distributed clusters, $400 \mu \mathrm{m}$ in diameter, arranged parallel to the pia mater. By contrast, the V/M of the EN (Case A, FC) did not differ significantly from unity at any field size suggesting a random distribution.

The spatial patterns exhibited by all histological features in the PSP cases as a whole are summarized in Table 2. The NFT, GI and TA were most frequently distributed at random or in regularly distributed clusters. By contrast, the EN and NP were largely randomly distributed. The surviving neurons and glial cell nuclei exhibited a random, regular, or clustered distribution in different regions. Contingency table analysis $\left(\chi^{2}\right)$ suggested that the NFT, GI, and TA were more frequently randomly distributed and less frequently uniformly distributed or aggregated into clusters compared with the surviving neurons and glial cell nuclei. The spatial patterns of some histological features varied in frequency in the cortex and $\mathrm{HC}$ compared with subcortical regions. Hence, NFT were more frequently clustered in the cortex and $\mathrm{HC}\left(\chi^{2}=7.64, \mathrm{P}<\right.$ $0.05)$ while $\mathrm{EN}$ were more frequently clustered in subcortical regions $\left(\chi^{2}=11.24 \mathrm{P}<\right.$ 
0.05). Surviving neurons were more frequently distributed in clusters in the cortex and $\mathrm{HC}$ but were more frequently randomly distributed in subcortical regions $\left(\chi^{2}=\right.$ 29.10, $\mathrm{P}<0.001$ ). There were no significant correlations between the frequency of specific types of spatial pattern or cluster size with disease onset, duration or Braak tangle score.

A summary of the spatial correlations between the densities of histological features is shown in Table 3. Variations in the density of NFT were not correlated with the densities of GI or TA. The densities of NFT, however, were positively correlated with the densities of surviving neurons and $\mathrm{EN}$ in $25 \%$ and $38 \%$ regions respectively. In addition, the densities of surviving neurons were positively correlated with the densities of EN in $39 \%$ of regions and negatively correlated with glial cell density in $27 \%$ of regions.

\section{Discussion}

The first objective was to determine the spatial patterns of the tau-immunoreactive inclusions in PSP and compare them with those reported previously in AD [40], PiD [41], and CBD [42]. In approximately $60 \%$ of brain regions in PSP, the NFT were randomly distributed and in only $25 \%$ of regions was there evidence of clustering of NFT, clustering being most evident in the cortex. In addition, the GI and TA were distributed in regular clusters in some subcortical regions but also randomly distributed in many regions while the EN and NP were most frequently randomly distributed. By contrast, in AD [40], PiD [41], and CBD [42] neuronal inclusions were frequently clustered, the clusters exhibiting a regular distribution parallel to the tissue boundary. Hence, the spatial patterns of inclusions in PSP differ from those previously studied in the tauopathies, being more frequently randomly distributed and less frequently regularly distributed in clusters.

Aging is likely to have several affects on the pathology of PSP [43] including effects on density and spatial patterns of inclusions. However, there were no significant correlations between the frequency of specific types of spatial pattern or cluster size with disease onset or duration. In addition, there were no significant correlations between spatial patterns and AD pathology as measured by Braak tangle score. AD 
and PSP have different spatial distributions of the pathology which may explain the lack of correlation. However, a much larger series of cases would be required to specifically test the hypothesis that patient age, duration of disease, or Braak score influences spatial pattern in PSP.

A regular distribution of clusters of NFT was most frequently observed in the cerebral cortex and in sectors $\mathrm{CA} 1 / 2$ of the HC. Cortical regions have a distinct anatomical structure, the major feature of which is the replicated local neural circuit (represented by 'columns' or 'modules') [44]. Various anatomical pathways connecting the cortex and the hippocampus also have a modular structure. The diameter of individual cortical modules varies between 500 and $1000 \mu \mathrm{m}$ depending on region [25,41] and those that comprise specific cortico-cortical pathways occur in bands that are more or less regularly distributed along the cortex. Previous studies of the tauopathies suggest that inclusions in the cortex may be associated with these projections [23]. Hence, the random distribution of NFT in subcortical regions could be attributable to a lack of a distinct modular organization. Nevertheless, the EN, GCI and AP were clustered in some subcortical regions. In addition, projections from the cortex to the cerebellum are topographically organized in columns [45] while in the striatum, corticostriate terminals originating from a particular area of cortex also exhibit a patchy stripe-like distribution [45]. An alternative explanation is that the pathology of PSP may develop initially in subcortical areas in association with specific anatomical pathways. However, as the disease develops the pathology becomes more widespread and diffuse so that the original topographic pattern is lost. By contrast, pathology in cortical areas may be a later development and more likely to retain evidence of the original topographic pattern of inclusions [6-8].

The second objective was to determine the spatial relationships between the histological features. As in the synucleinopathy multiple system atrophy (MSA), there is prominent glial cell pathology in PSP and the relationship between the neuronal and glial cell pathologies in PSP has not been established. Glial pathology could be secondary to that of the neuronal pathology, be the primary pathological change, or occur independently but concurrently with neuronal pathology. In PSP, there were few spatial correlations between the densities of NFT and the GI, TA, or glial cell nuclei within individual regions. The NFT, however, were often more widely 
distributed and abundant than both the GI and TA [8] suggesting that neuronal pathology is the primary change in PSP. Hence, the lack of spatial correlation observed between neuronal and glial cell pathologies in specific regions may be a consequence of the more widespread distribution of the NFT and the more localized clustering of the GI and TA.

A positive spatial correlation was observed between the densities of NFT and EN in approximately $40 \%$ of brain regions studied. In PSP, EN are less abundant compared with similar cellular changes in PiD ('Pick' cells) [17] or CBD ('ballooned neurons') [17] but were more frequent in subcortical areas and often developed in large clusters in the pontine nuclei and inferior olivary nucleus [43]. There are two possible explanations for this correlation. First, NFT and EN could represent different stages of the same pathology, i.e., neuronal enlargement may precede or follow that of the development of NFT. Nevertheless, few EN had NFT and neurons containing NFT were not noticeably enlarged in our cases. Second, NFT and EN could represent different pathological processes occurring independently but within the same columns of neurons. Hence, NFT and EN could be the consequence of disruption of the cytoskeleton and the effect of synaptic disconnection respectively. Lack of correlation between NFT and EN in a significant proportion of regions could be attributable to the 'stage' of the disease, i.e., as the disease develops and pathology becomes more widespread, the correlation is lost. However, positive correlations between NFT and EN were not significantly more frequent in shorter duration cases which would argue against this hypothesis.

Previous data suggest that several types of NCI, including those in PSP, regardless of morphology or molecular composition, are clustered in the cortex and, in a significant proportion of gyri, the clusters of NCI are regularly distributed parallel to the pia mater [23]. The spatial patterns of the NCI is consistent with their development in association with the cells of origin of specific anatomical pathways [23] and therefore could be the result of cell to cell transfer of pathogenic proteins proposed by Goedert et al [46] and Steiner et al [47]. In addition, in many neurodegenerative disorders, the density of pathological inclusions varies significantly perpendicular to the pia mater [8]. The laminar distribution of an inclusion may reflect degeneration of neural pathways that have their cells of origin or axon terminals located within one or more 
laminae and can therefore also indicate the pattern of cortical degeneration in a disorder.

In conclusion, the cellular inclusions in PSP exhibit a different spatial pattern compared with other disorders with tau-immunoreactive inclusions such as AD [40], PiD [41], and CBD [42]. NFT were frequently randomly distributed in PSP especially in subcortical areas, consistent with a widespread neuronal pathology and which is not restricted to a specific anatomical pathway. In cortical regions, however, the NFT exhibit a spatial pattern similar to that observed in other tauopathies, e.g., a regular distribution of clusters parallel to the tissue boundary consistent with a later development. GI and TA remain more localized in their distribution and may retain their original regular pattern of clustering in subcortical areas. The data also suggest that there is a spatial correlation between the NFT and EN at some stages of the disease.

\section{Acknowledgements}

We would like to thank the Department of Neuropathology, Institute of Psychiatry, King's College London for supplying the cases of PSP and Heidi Barnes and Mavis Kibble for their excellent technical assistance. The authors report no conflict of interest.

\section{References}

1. Steele JC, Richardson JC, Olszewski T (1964) Progressive supranuclear palsy. Arch Neurol 10: 333-359.

2. Schrag A, Ben-Shlomo Y, Quinn NP (1999) Prevalence of progressive supranuclear palsy and multiple system atrophy: a cross-sectional study. Lancet 354:1771-1775.

3. Nath U, Ben-Shlomo Y, Thomson RG, et al (2001) The prevalence of progressive supranuclear palsy (Steele-Richardson-Olszewski syndrome) in the UK. Brain 124: 1438-1449. 
4. Papapetropoulos S, Gonzalez J, Mash DC (2005) Natural history of progressive supranuclear palsy: a clinicopathologic study from a population of brain donors. Eur Neurol 54: 1-9.

5. Lantos PL (1994) The neuropathology of progressive supranuclear palsy. J Neural Transm (Suppl.) 42: 137-152.

6. Gomez-Haro C, Espert-Torbajada R, Gadea-Domenech M, Navarro-Humanes JF (1999) Progressive supranuclear palsy: Neurological, neuropathological and neuropsychological aspects. Rev de Neurol 9: 936-956.

7. Armstrong RA, Lantos PL, Cairns NJ (2009a) Hippocampal pathology in progressive supranuclear palsy (PSP): a quantitative study of 8 cases. Clin Neuropathol 28: 46-53.

8. Armstrong RA, Cairns NJ (2009b) Laminar distribution of the pathological changes in frontal and temporal lobes in eight patients with progressive supranuclear palsy. Clin Neuropathol 28: 350-357.

9. Cordata NJ, Duggins AJ, Halliday GM, Morris JGL, Pantelis C (2005) Clinical deficits correlate with regional cerebral atrophy in progressive supranuclear palsy. Brain 128: 1259-1266.

10. Lantos PL (1994) The neuropathology of progressive supranuclear palsy. J Neural Transm (Suppl.) 42: 137-152.

11. Daniel SE, Bebruin VMS, Lees AJ (1995) The clinical and pathological spectrum of Steele-Richardson-Olszewski syndrome (Progressive supranuclear palsy)- a reappraisal. Brain 118: 759-770.

12. Tellez-Nagel I, Wisniewski HM (1973) Ultrastructure of neurofibrillary tangles in Steele-Richardson-Olszewski syndrome. Arch Neurol 29: 324-327. 
13. Montpetit V, Clapin DF, Guberman M (1985) Substructure of 20nm filaments of progressive supranuclear palsy. Acta Neuropathol 68: 311-318.

14. Dickson DW (1999) Neuropathological differentiation of progressive supranuclear palsy and corticobasal degeneration. J Neurol 246: 6-15.

15. Dickson DW (2003) Neurodegeneration: The Molecular Pathology of Dementia and Movement Disorders. International Society for Neuropathology (ISN) Press, Basel, Switzerland.

16. Cairns NJ, Lee VM-Y, Trojanowski JQ (2004) The cytoskeleton in neurodegenerative disease. J Pathol 204: 438-449.

17. Mackenzie IRA, Hudson LP (1995) Achromatic neurons in the cortex of progressive supranuclear palsy. Acta Neuropathol 90: 615-619.

18. Yamada T, McGeer PL, McGeer EG (1992) Appearance of paired nucleated taupositive glia in patients with progressive supranuclear palsy brain tissue. Neurosci Lett 135: 99-102.

19. Ikeda K, Akiyama H, Kondo H, et al (1995) Thorn-shaped astrocytes: possibly secondarily induced tau-positive glial fibrillary tangles. Acta Neuropathol 90: 620625 .

20. Komori T (1999) Tau positive glial inclusions in progressive supranuclear palsy, corticobasal degeneration and Pick's disease. Brain Pathol 9: 663-679.

21. Litvan I, Grimes DA, Lang AE, Jankovic J, McKee A, Verny M, Jellinger K, Chaudhuri KR, Pearce RKB (1999) Clinical features differentiating patients with postmortem confirmed progressive supranuclear palsy and corticobasal degeneration. J Neurol 246: 1-5. 
22. Arima K, Nakamura M, Sunohara N, et al (1999) Immunohistochemical and ultrastructural characterization of neuritic clusters around ghost tangles in the hippocampal formation in progressive supranuclear palsy. Acta Neuropathol 97: 565576.

23. Armstrong RA, Cairns NJ, Lantos PL (2001) What does the study of spatial patterns tell us about the pathogenesis of neurodegenerative disorders? Neuropathology 21: 1-12.

24. De Lacoste M, White CL III (1993) The role of cortical connectivity in Alzheimer's disease pathogenesis: a review and model system. Neurobiol Aging 14: $1-16$.

25. Hiorns RW, Neal JW, Pearson RCA, Powell TPS (1991) Clustering of ipsilateral cortico-cortical projection neurons to area 7 in the rhesus monkey. Proc R Soc Lond 246: $1-9$.

26. Armstrong RA, Lantos PL, Cairns NJ (2007a) Spatial topography of the neurofibrillary tangles in cortical and subcortical regions in progressive supranuclear palsy. Parkinsonism Rel Disord 13: 50-54.

27. Litvan I, Agid Y, Calne D, et al (1996a) Clinical research criteria for the diagnosis of progressive supranuclear palsy (Steele-Richardson-Olszewski syndrome): report of the NINDS-SPSP International Workshop. Neurology 47: 1-9.

28. Litvan I, Hauw JJ, Bartko JJ, et al (1996b) Validity and reliability of the preliminary NINDS neuropathological criteria for progressive supranuclear palsy and related disorders. J Neuropath Exp Neurol 55: 97-105.

29. Hauw JJ, Daniel SE, Dickson D, et al (1994) Preliminary NINDS neuropathologic criteria for Steele-Richardson-Olszewski syndrome (PSP). Neurology 44: 2015-2019. 
30. Williams DR, Holton JL, Strand K, de Silva R, Lees AJ, Revesz T (2007) Differences in tau load are associated with different clinical phenotypes in progressive supranuclear palsy. Neuropathol Appl Neurobiol 33: 256-257.

31. Braak H, Braak E, Bohl J (1993) Staging of Alzheimer-related cortical destruction. Eur Neurol 33: 403-408.

32 Mattila P, Togo T, Dickson DW (2002) The subthalamic nucleus has neurofibrillary tangles in argyrophilic grain disease and in advanced Alzheimer's disease. Neurosci Lett 320: 81-85.

33. Hattori M, Hashizume Y, Yoshida M, Iwasaki Y, Hishikawa N, Ueda R, Ojika K (2003) Distribution of astrocytic plaques in corticobasal degeneration brain and comparison with tuft shaped astrocytes in the progressive supranuclear palsy brain. Acta Neuropathol 106: 143-149.

34. Aoki K, Uchihara T, Nakamura A, Komori T, Arai N, Mizutani T (2003) Expression of apolipoprotein $\mathrm{E}$ in ballooned neurons: comparative immunohistochemical study in neurodegenerative disorders and infarctions. Acta Neuropathol 106: 436-440.

35. Fujino Y, Delucia MW, Davies P, Dickson DW (2004) Ballooned neurons in the limbic lobe are associated with Alzheimer-type pathology and lack diagnositic specificity. Neuropathol Appl Neurobiol 30: 676-682.

36. Minamu M, Mizutani T, Kawanishi R, Suzuki Y, Mori H (2003) Neuronal expression of alpha B crystalline in cerebral infarction. Acta Neuropathol 105: 549554.

37. Armstrong RA (1993a) The usefulness of spatial pattern analysis in understanding the pathogenesis of neurodegenerative disorders with special reference to plaque formation in Alzheimer's disease. Neurodegeneration 2: 73-80. 
38. Armstrong RA (2006) Methods of studying the planar distribution of objects in histological sections of brain tissue. J Microsc 221: 153-158.

39. Armstrong RA (2003) Measuring the degree of spatial correlation between histological features in thin sections of brain tissue. Neuropathology 23: 245-253.

40. Armstrong RA (1993b) Is the clustering of neurofibrillary tangles in Alzheimer's disease related to the cells of origin of specific cortico-cortical projections? Neurosci Lett 160: 57-60.

41. Armstrong RA, Cairns NJ, Lantos PL (1998) Clustering of Pick bodies in Pick's disease. Neurosci Lett 242: 81-84.

42. Armstrong RA, Cairns NJ, Lantos PL (1999) Clustering of cerebral cortical lesions in patients with corticobasal degeneration. Neurosci Lett 268: 5-8.

43. Armstrong RA, Lantos PL, Cairns NJ (2007b) Progressive supranuclear palsy (PSP): a quantitative study of the pathological changes in cortical and subcortical regions of eight cases. J Neural Transm 114: 1569-1577.

44. Mountcastle VB (1979) An organizing principle for cerebral function: The unit module and the distributed system. In: The Neurosciences. $4^{\text {th }}$ Study Program. Ed FO Schmitt and FG Worden, pp. 21-42.

45. Brodal A (1981) Neurological Anatomy in relation to Clinical Medicine, $3^{\text {rd }}$ Ed., New York and Oxford: Oxford University Press.

46. Goedert M, Clavaguera F, Tolnay M (2010) The propagation of prion-like protein inclusions in neurodegenerative diseases. Trends in Neurosciences 33: 317-325.

47. Steiner JA, Angot E, Brunden P (2011) A deadly spread: cellular mechanisms of $\alpha$-synuclein transfer. Cell Death and Different 18: 1425-1433. 
Table 1. Demographic features, gross fresh brain weight, and Braak stage of the eight progressive supranuclear palsy cases (PSP) studied.

\begin{tabular}{llllllll}
\hline Case & Sex & Onset & Age & Atrophy & BW & NP & Braak stage \\
& $(\mathrm{yrs})$ & $(\mathrm{yrs})$ & & & & & \\
& & & & & & & \\
A & F & 53 & 64 & general, Fr & 961 & + & 1 \\
B & M & 65 & 70 & none & $1175+$ & 1 \\
C & M & 66 & 74 & Mo, Fr,P & $498 \mathrm{R}$ & 0 & 2 \\
D & M & 59 & 66 & none & $1016+$ & 1 \\
E & F & 69 & 75 & Mo, Fr,P & $515 \mathrm{R}+$ & 1 \\
F & M & 76 & 86 & mild & $1063 \quad 0$ & 3 \\
G & F & 75 & 81 & none & $1037++$ & 2 \\
H & F & 67 & 71 & slight Fr,P & $562 \mathrm{R}$ & 0 & 1 \\
& & & & & & & \\
\hline
\end{tabular}

Abbreviations: $\mathrm{M}=$ Male, $\mathrm{F}=$ Female, $\mathrm{Fr}=$ Frontal lobe, $\mathrm{P}=$ Parietal lobe, $\mathrm{Mo}=$ Moderate, $\mathrm{R}=$ Right hemisphere, $\mathrm{BW}=$ Brain weight, Neuritic plaque (NP) density: 0 $=$ absent,$+=$ few,$++=$ moderate. 
Table 2. Frequency of the different types of spatial pattern in cortical and subcortical areas in eight cases of progressive supranuclear palsy.

\begin{tabular}{llllll}
\hline Feature & Region & R & Reg & Regular clusters & Large clusters \\
\hline NFT & Cortex/HC & 11 & 0 & 9 & 2 \\
& Subcortex & 29 & 1 & 5 & 1 \\
GI & Cortex/HC & 4 & 0 & 5 & 2 \\
& Subcortex & 18 & 1 & 12 & 2 \\
TA & Cortex/HC & 3 & 0 & 0 & 0 \\
& Subcortex & 4 & 0 & 7 & 2 \\
NP & Cortex/HC & 4 & 0 & 0 & 0 \\
& Subcortex & 0 & 0 & 0 & 0 \\
EN & Cortex/HC & 23 & 0 & 3 & 1 \\
& Subcortex & 10 & 2 & 2 & 7 \\
SN & Cortex/HC & 12 & 33 & 5 & 17 \\
& Subcortex & 33 & 6 & 7 & 19 \\
GL & Cortex/HC & 23 & 17 & 9 & 6 \\
& Subcortex & 14 & 16 & 6 &
\end{tabular}

Abbreviations: $\mathrm{R}=$ Random distribution, $\mathrm{Reg}=$ Regular distribution, $\mathrm{NFT}=$ Neurofibrillary tangles, GI $=$ Glial inclusions, $\mathrm{TA}=$ Tufted astrocytes, $\mathrm{NP}=$ Neuritic plaques, $\mathrm{EN}=$ Enlarged neurons, $\mathrm{SN}=$ Surviving neurons, $\mathrm{GL}=$ Glial cell nuclei, $\mathrm{HC}$ = Hippocampus.

Chi-square $\left(\chi^{2}\right)$ contingency table tests: Statistically significant comparisons only: 1) Between different lesions totaled over all regions: NFT/SN $\chi^{2}=41.68 * * *$, NFT/GL $\chi^{2}=38.98^{* * *}, \mathrm{GI} / \mathrm{EN} \chi^{2}=9.40^{*}, \mathrm{GI} / \mathrm{SN} \chi^{2}=29.85^{* * *}, \mathrm{GI} / \mathrm{GL} \chi^{2}=25.05^{* * *}$, TA/EN $\chi^{2}=12.43 * *$ TA/SN $\chi^{2}=19.15^{* * *}$, TA/GL $\chi^{2}=14.57 * *$, EN/SN $\chi^{2}=$ $31.01 * * *, \mathrm{EN} / \mathrm{GL} \chi^{2}=31.45^{* * *} ; 2$ ) Comparison of individual lesions between cortex/HC and subcortical regions; NFT $\chi^{2}=7.64(3 \mathrm{DF}, \mathrm{P}<0.05)$, EN $\chi^{2}=11.24$ (3DF, $\mathrm{P}<0.05)$; $\mathrm{SN} \chi^{2}=29.10$ (3DF, $\left.\mathrm{P}<0.001\right)$. All other comparisons were not statistically significant. 
Table 3. Frequency of the spatial correlations (Pearson's ' $r$ ') between lesions, in cortical and subcortical areas in progressive supranuclear palsy (PSP).

\section{Frequency of correlation}

\begin{tabular}{|c|c|c|c|c|}
\hline Variables correlated & $\mathrm{N}$ & Positive ' $r$ ' & Negative 'r' & NS \\
\hline NFT/GI & 49 & 4 & 2 & 43 \\
\hline NFT/TA & 16 & 1 & 0 & 15 \\
\hline NFT/EN & 38 & 15 & 0 & 23 \\
\hline NFT/SN & 68 & 17 & 0 & 51 \\
\hline NFT/GL & 71 & 5 & 7 & 59 \\
\hline GI/TA & 18 & 0 & 0 & 18 \\
\hline GI/EN & 31 & 2 & 0 & 29 \\
\hline GI/SN & 59 & 0 & 6 & 53 \\
\hline GI/GL & 58 & 5 & 0 & 53 \\
\hline TA/EN & 9 & 4 & 0 & 5 \\
\hline $\mathrm{TA} / \mathrm{SN}$ & 20 & 1 & 0 & 19 \\
\hline TA/GL & 20 & 1 & 2 & 17 \\
\hline $\mathrm{EN} / \mathrm{SN}$ & 61 & 24 & 1 & 36 \\
\hline EN/GL & 60 & 4 & 3 & 53 \\
\hline SN/GL & 119 & 0 & 33 & 86 \\
\hline
\end{tabular}

Abbreviations: NFT $=$ Neurofibrillary tangles, $\mathrm{GI}=$ Glial inclusions, $\mathrm{TA}=$ Tufted astrocytes, EN = Enlarged neurons, $\mathrm{SN}=$ Surviving neurons, GL = Glial cell nuclei, $\mathrm{N}=$ total number of brain regions analyzed, $\mathrm{NS}=$ no significant correlation. Neuritic plaques were present in too few regions and at too low a density to test spatial correlations. 


\section{Legends to figures}

Fig 1. Neurofibrillary tangles (NFT) (arrow) in the midbrain of a case of progressive supranuclear palsy (PSP) (tau immunohistochemistry, haematoxylin, bar $=10 \mu \mathrm{m})$

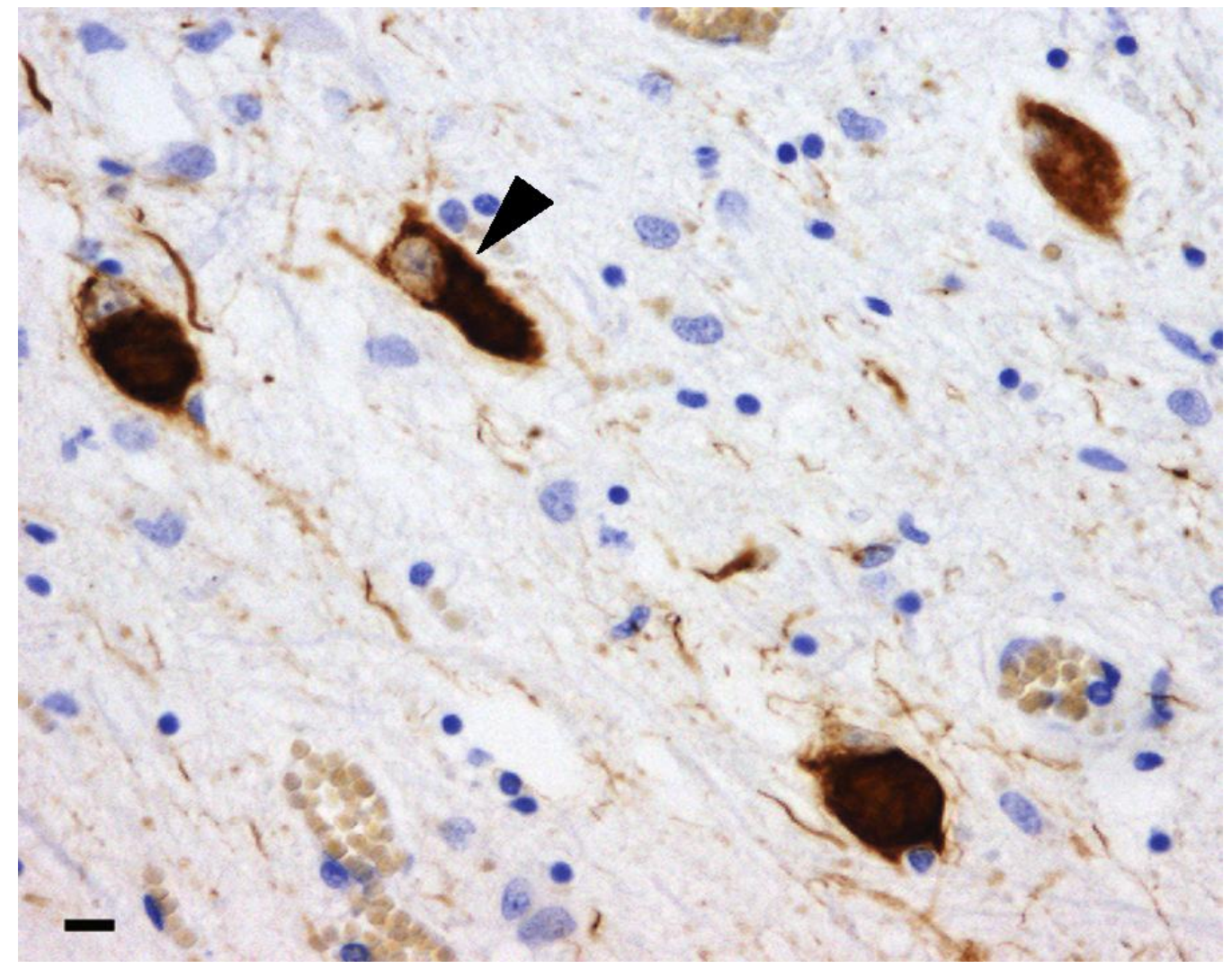


Fig 2. 'Tufted' astrocytes (TA) (arrow) in the putamen of a case of progressive supranuclear palsy (PSP) (tau immunohistochemistry, haematoxylin, bar $=10 \mu \mathrm{m}$ ).

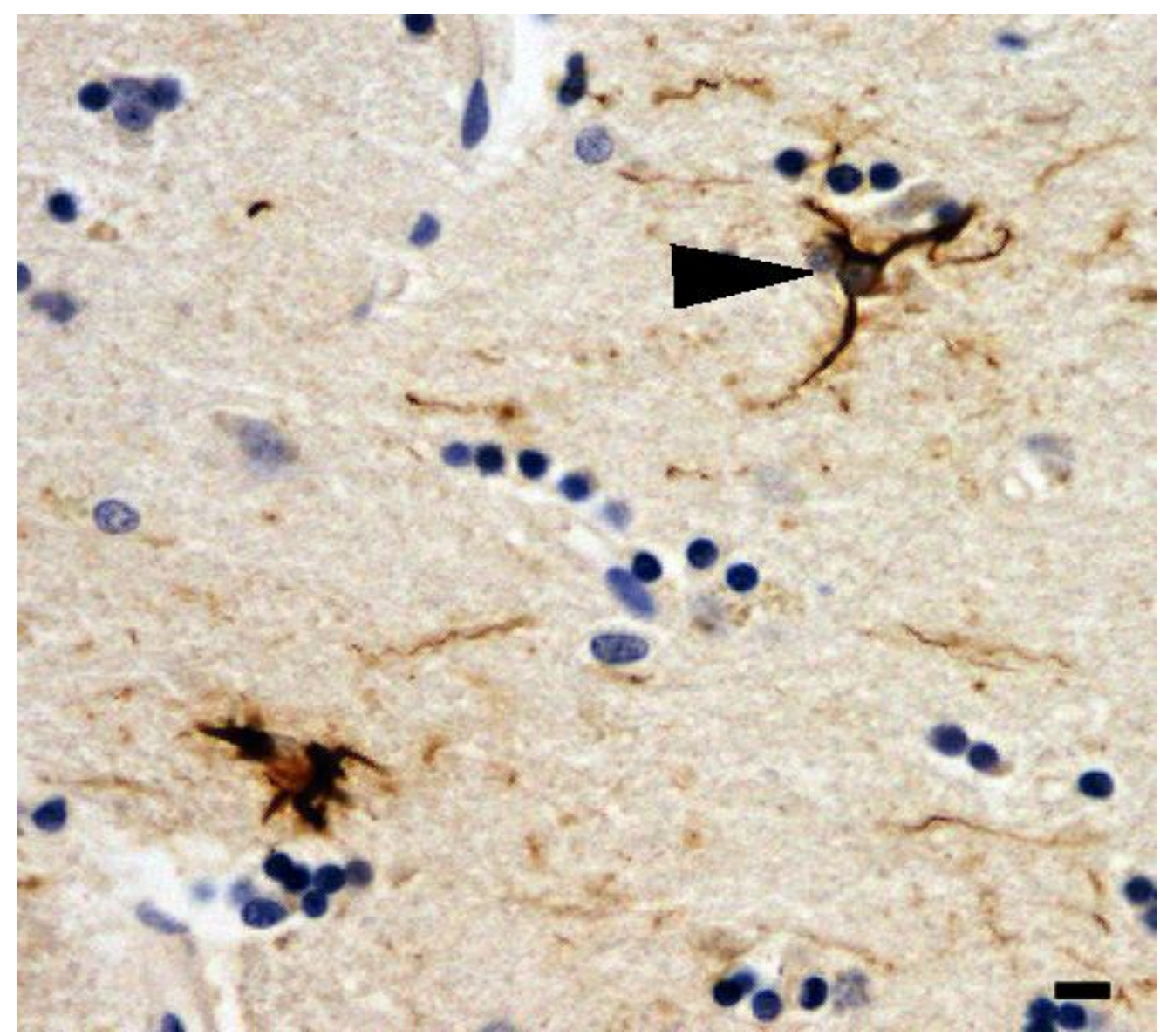


Fig 3. Examples of the spatial patterns exhibited by the neurofibrillary tangles (NFT), and enlarged neurons (EN) in the cerebral cortex in a case of progressive supranuclear palsy (PSP) (* significant V/M peak).

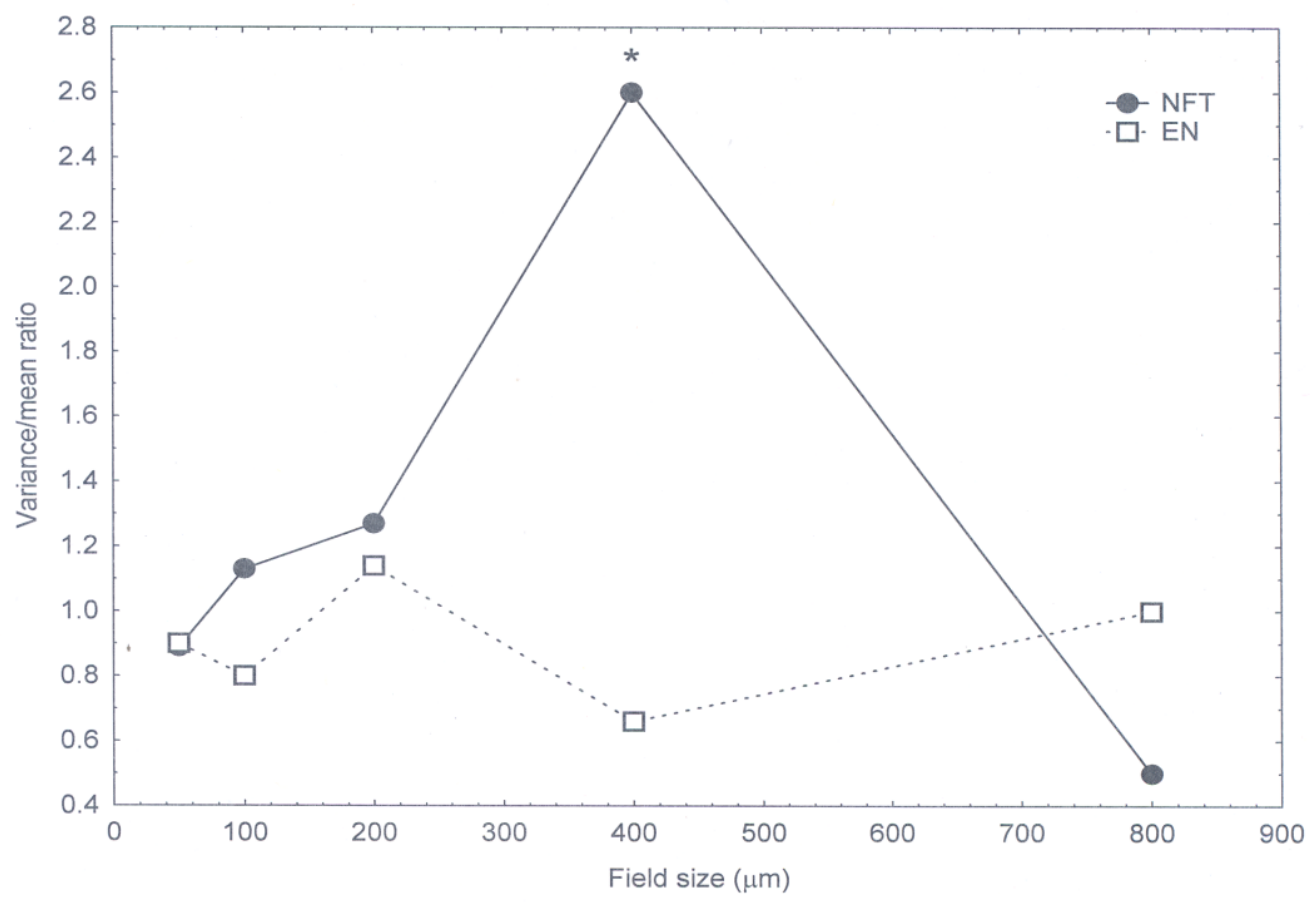

